\author{
Military Technical College \\ Kobry El-Kobbah, \\ Cairo, Egypt.
}

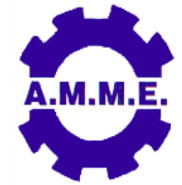

$18^{\text {th }}$ International Conference on Applied Mechanics and Mechanical Engineering.

\title{
INVESTIGATING WEAR RESISTANCE OF LOW CARBON STEELS REINFORCED BY SIC SUBMICRON SIZED PARTICLES
}

\author{
T. Mattar ${ }^{1}$, M. K. El-Fawakhry ${ }^{2}$ and I. El-Mahallawi ${ }^{3}$
}

\begin{abstract}
The development of new steel grades with improved mechanical properties by the addition of nanoparticles to microalloyed steels has recently gained attention. Misfit, size, thermal expansion coefficient, density, wettability and stability were reported to have an effect on the properties. The main challenge is the development of these nanostructured constituents by modified conventional and advanced manufacturing techniques. This work discusses the options for applying nanoinoculation techniques to produce new nanodispersed steel grades, with improved mechanical properties. At this stage of work submicron sized particles coated with $\mathrm{Cu}$ were selected in order to avoid wettability and agglomeration problems. The inoculant was added in different ratios starting from $0.025 \%$ up to $0.10 \%$ to the steel melt and the stirring of the melt was secured via the induction current heating for 4 minutes. The produced alloy was tapped in an iron ingot, and then hot forged into bars and plates. Optical microscopic studies were performed on the new material in order to identify the microstructural features. The wear abrasion resistance was detected using a wear test. It is shown that these new steel grades have improved wear friction coefficients; though higher wear rates at the studied load and time conditions. This opens a new path for investigations on the wear resistance of nanodispersed and nanoinoculated steels.
\end{abstract}

1 Professor and President of Centre of Metallurgical Research and Development Institute CMRDI. tahamattar@yahoo.com. 01001301230.

2 Lecturer, Centre of Metallurgical Research and Development Institute CMRDI. mohamedelfawkhry@gmail.com; abdrabu1979kamal@yahoo.com. 01152529685.

3 Professor and Head of Mining, Petroleum and Metallurgical Engineering Department, Faculty of Engineering, Cairo University. ielmahallawi@bue.edu.eg; saiman@eng1.cu.edu.eg. 01006044661. 


\section{INTRODUCTION}

\section{The Wear Phenomenon}

The wear phenomenon consists of the removal of material from the surfaces of one of the contacting bodies through inter action with other contacting bodies. Although it is probable that numerous processes exist in metal sliding situations, the following four are stressed [1]: 1) metal transfer, 2) film formation and removal, 3) debris generation, and 4) cycle surface deterioration. The applicability of these processes to the induction of sudden transitions in friction or wear rate depends greatly on the sliding material, the contact geometry, the thermal and chemical environment of the contact and the mechanical parameters of the system; such as the level of stress. Numerous [1,2] examples exist for instances of sudden catastrophic changes in wear rate due to debris generation.

The physical properties of materials, which determine the wear resistance of materials, include hardness; transverse rupture strength; impact toughness and heat resistance. Various investigations have shown that microstructure [3] strongly influences wear resistance, together with alloy composition as part of the tribological system. Previous results [5-8] have also shown that hardness is not a suitable parameter to describe reliably the wear resistance of inhomogeneous or multiphase microstructures. The microstructure- hardness - wear relationships were emphasized to be important $[4,5]$.

Earlier studies [1,9] made it possible to identify four major types of wear: 1) adhesive wear, 2) abrasive wear, 3) corrosive wear, and 4) Surface fatigue wear. In many cases, two or more types of wear occur simultaneously. Apart from these four major types of wear, other forms include the following: fretting wear, fretting corrosion, erosive wear, cavitation wear, chipping, Oxidative wear, and thermal wear. The effect of contact stresses on abrasive wear is an important factor to be considered. High repetitive contact forces between a surface and abrasive lead to plastic deformation and fracture. Impact properties should also be considered [10], as some components are subjected to impact as well as abrasion. Investigations [11-12] revealed microstructure to be a key factor in the impact wear resistance which is also required in tooling alloys involving sliding wear and it was concluding [11] that the volume fraction of marten site and ferrite in duplex microstructures is an important factor in wear resistance.

\section{Metallurgical Factors Affecting the Wear Resistance of Steels}

Low carbon steels (low C steels) are widely used in structural engineering applications in the petroleum and energy fields due to their good strength and ductility with economic competence. Some of these applications would include harsh environments, which lead to failure of these parts due to incompetence of their wear resistance. Developing new generations of low C steels with enhanced wear resistance would improve the life of such structures.

A study [1] of the metallurgical factors affecting adhesive wear resistance attributed the practical problem with adhesive wear to minimizing the frequency and scale of metal displacement. Steels resisting oxidation or steels operating in an inert environment were reported to be more likely to wear by a severe mechanism (as 
oxidized surface resist further wear). Discontinuous microstructure were found to be an advantage in adhesive wear, homogeneous single phase materials being prone to excessive metal displacement and tearing, especially if the grain size is large. In this respect, elements dissolving in solid solution will not improve adhesive wear resistance and will possibly even lower it. The quantity of carbide formed is a deciding factor in adhesive wear resistance. In low alloy steels [1], the effect of alloying elements depends on their carbide forming tendency and their solubility in ferrite. Elements, which dissolve to produce solid solution hardening, have very little influence on wear resistance.

Enhancing wear by forming particle reinforced metal matrix composites has gained wide interest during the last decades. Under appropriate conditions, the addition of ceramic particles to a metal matrix can lead to improved wear resistance. It should be recognized, however, that if the conditions are not suitable, the addition of ceramic particles might decrease wear resistance. For example, the ceramic particles may be removed from the matrix and lead to a wear process by three body abrasive wear. Another point to keep in mind is that as the volume fraction or size of the reinforcement becomes large, the fracture toughness of the composite will be reduced significantly. If the fracture toughness is inadequate, the particles will fracture and contribute to the wear process. Increasing wear resistance without a penalty in toughness is a challenge [13].

\section{Role of Nanoparticles in Improving Mechanical Properties and Wear Resistance of Steels}

New steel grades with improved mechanical properties are developed by the addition of nanoparticles to microalloyed steels. Misfit, size, thermal expansion coefficient, density, wettability and stability were reported to have an effect on the properties. The aim of the addition of nanoparticles to the microalloying steel grades is to increase the tensile strength and the fatigue limit, for power train components such as hot forged crankshafts and connecting rods. On the other hand, another aim of the addition of nanoparticles is to increase the toughness without decreasing the tensile strength of some steering components manufactured by cold forging [14].

Though, literature contains information on the role of nanodispersion on the wear resistance of aluminum alloys [15, 16], scarce information exists on the wear resistance of steels, though, the presence of nanometric precipitates was reported to result an improvement of the yield strength value of the API steel [17]. The obtained yield strength value was $860 \mathrm{MPa}$ and the strengthening effect was attributed to solid solution strengthening, grain size effect and dislocation hardening. Silicon carbide powder covering the size range of micron, sub-micron and nano-scale was used [18] as nanoinoculant in melting and production of low alloy steel used for Tanks and containers.

Recent studies [18, 19] adopt a new technology to enhance the wear resistance of steels by adding nanoparticles as a reinforcement to the matrix. The proposed technology is based on the modification of the microstructure by using low cost nanosized additions, which leads to significant increase and improvement in all the properties of steel as a result of eliminating heterogeneity in the composition, shape and size of the steel, which allows the use of these modified steels in advanced applications. Inoculant technique is considered among the recent methods to 
produce ultrafine and nanostructured steels. The main challenge in this technology is to obtain a uniform and homogenous dispersion for the nano-sized ceramic particles in the steel matrix when using the conventional casting technique.

The effect of nanocrystalline sizes on the mechanical properties of materials by a variety of computer simulation models based on molecular dynamics was reviewed by Julia R. Weertman [20]. This compiled work opened many questions to the beneficial role of nanostructuring on materials engineering applications when mechanical properties are sought, as the models predicted enhanced strengthening, but failed to make sound predictions for plastic deformation behaviour. However, it was shown that nanocrystalline metals have a promising potential if an internal structure that produces adequate strain hardening to prevent early plastic instabilities is devised.

Previous studies have, however, shown discrepancy in wear performance of nanodispersed material prepared by different techniques and have attributed wear to indentation-induced sub-surface mechanism [21, 22], powder characteristics, the extent of reaction and decarburization during spraying, and the resultant microstructure in the coating during rapid solidification of the particles at high cooling rates [22]. It was also shown that the physical properties of the coatings produced from the nanocrystalline powders differ noticeably from those of the conventional powders [23]. The associated grain size refinement processes not only reduce the grain structure of the powders, but also increase the surface area of the powder particles. Nanostructured coatings have been predicted to provide solutions to improve on existing applications or to allow for new applications that require the unique and added benefits in engineering applications [24-26].

The previous review opens a new path for investigations on the wear resistance of nano-dispersed and nanoinoculated steels. The main challenge is the development of these nanostructured constituents by modified conventional and advanced manufacturing techniques.

\section{EXPERIMENTAL WORK}

Low carbon alloyed steel of the composition shown in Table 1 was prepared using coreless spinel crucible induction furnace. In this work, particles $(400-600 \mathrm{~nm}$ size) of $\mathrm{SiC}$ and copper-coated $\mathrm{SiC}$ of variant amounts (as shown in Table 2) were used. Silicon carbide powder covering the size range of micron, sub-micron and nano-scale was used [18] as nano inoculant in melting and production of low alloy steel used for this study. Submicron sized particles were chosen in this work to avoid the problems of agglomeration associated with nanoparticles. The addition of the submicron particles took place after complete melting of the charge, at a clean melt surface after full slag-off regime. This was aided by the stirring action resulting from the magnetic current of the induction furnace. The induction furnace was kept in operation after the addition for 4-5 minutes to ensure complete and uniform distribution of the added submicron sized particles into the molten pool. The molten metal was poured into a metal mold with dimensions of $\varphi 100 \mathrm{~mm}$, height $200 \mathrm{~mm}$. The produced ingots were forged at $1100^{\circ}$, and the finishing temperature was $1000^{\circ}$. Samples were taken from the square cross section bars for microstructure and hardness examination and wear test. The inoculant was added in different ratios starting from $0.025 \%$ up to $0.1 \%$ to 
the steel melt and the stirring of the melt was secured via the induction current heating for 4 minutes. Two sets of samples were prepared one with $\mathrm{SiC}$ and the other using Cu-coated $\mathrm{SiC}$ (for enhancing the wettability of the submicron sized particles with the molten steel). The $\mathrm{SiC}$ particles were coated with $\mathrm{Cu}$ because $\mathrm{Cu}$ melts at a lower temperature than steel and SiC and it forms a solid solution with $\gamma$ Fe. Therefore, the aim was to investigate the effect of creating a solid solution region around the $\mathrm{SiC}$ on the wettability between the austenite matrix and the $\mathrm{SiC}$.

Optical microscopic studies were performed on the new material in order to identify the microstructural features. The wear abrasion resistance was detected using a wear test. Wear tests were carried out using a pin-on-disc bench type setup using a $180 \mathrm{~mm}$ diameter hardened steel disc (C62). The hardness of the disc was $263 \mathrm{BHN}$, with an initial surface roughness of $\mathrm{Ra} 4.5 \mu \mathrm{m}$. Standard specimens with diameter of $8 \mathrm{~mm}$ and $20 \mathrm{~mm}$ length were prepared, inserted in the holder against the disc and loaded vertically. The wear tests were then performed for the cast material with the following parameters: velocity $=0.8 \mathrm{~m} / \mathrm{s}(250 \mathrm{rpm})$, time $=900 \mathrm{~s}$ and load $=5 \mathrm{~N}$. The \%age of differences in the weight of the samples were taken as an indication of the wear resistance of the material.

\section{RESULTS AND DISCUSSION}

Figure 1 shows the effect of adding submicron sized $\mathrm{SiC}$ particles on obtaining increased pearlite content. The optical microscopic studies showed that the fraction of pearlite, as well as its fineness depends on the percentage of SiC inoculation. The increase and enhancement of the pearlite structure associated by increasing the percentage of $\mathrm{SiC}$ inoculants was attributed to the dissociation of $\mathrm{SiC}$ at high temperature due to the formation of iron silicide and carbon [18]. This free carbon reduces the free energy of the austenite decomposition into pearlite, and then the volume fraction of pearlite is increased with increasing the percentage of $\mathrm{SiC}$ inoculants. In the previous work by the authors, it was reported that the mechanical properties of the new steels were enhanced. It was shown that impact toughness of inoculated steel approached $250 \%$ more than the non-inoculated steel. The change in volume fraction of pearlite was associated by changes in the tensile strength [18]. The improvement in wear abrasion resistance was observed in the earlier work for $\mathrm{SiC}$ additions exceeding $0.1 \%$ (Fig.2). The refined grain size and increased pearlite content, combined with the enhanced mechanical properties explained the improved wear resistance of the submicron sized dispersed steels.

The effect of submicron and nanoparticles addition to metallic alloys is interpreted by understanding the role of nanodispersions on the microstructure and mechanical properties needs further investigation, as it is well known that both microstructure and hardness determine the wear resistance of steels. A new model has been presented that considers [25] that the high mechanical resistance of nanoreinforced composites is the result of the incremental summation of several strengthening mechanism contributions, namely: load transfer effect, Hall-Petch strengthening, Orowan strengthening, coefficient of thermal expansion (CTE) and elastic modulus (EM) mismatch. However, knowledge on the behavior and properties of the interface at the boundary of the two different materials (nanomodifier - liquid/solidifying metal) is still at a premature state. Models describing the interface of nanomodifier - alloy need to be created and solved by means of numerical methods. 
Figure 3 shows the microstructure of the material prepared for this work. It is shown from the figure that adding the submicron sized particles to the steel has resulted a significant refining effect on the grain size (from about $50 \mu \mathrm{m}$ to $30 \mu \mathrm{m}$ ). The effect of Cu-coating was not reflected in the microstructure investigations. The refining effect may be explained by the role of inoculants as nucleating sites, which has been achieved as a result of the improved addition technology adopted in this work, by letting the melt to stir in the furnace for 5 minutes. The nanoparticles tend to agglomerate if their addition technique is not well controlled. The refined microstructure of the steel is expected to result enhancement in the mechanical properties, as previously reported in the authors previous study [18]. When the grain size decreases, the yield strength (YS) as well as the ultimate tensile strength (UTS) increases, according to the well-known Hall-Petch effect [27].

Figure 4 shows the obtained wear test results, from which it is shown that sporadic results are obtained for the wear resistance of the nano-inoculated steel. The figure shows that the addition of the submicron sized particles in the range of $0.025 \%$ does not result changes in the wear\% of the steel, whereas, further addition result deterioration in the wear resistance of the steel. The increase of the wear\% of T13 with $0.05 \%$ submicron sized particles of $\mathrm{SiC}$ is not explained. However, the wear results of the $\mathrm{Cu}$-coated $\mathrm{SiC}$ particles show deterioration in the wear resistance of the steel, for all \%ages less than $0.1 \%$ and a slight decrease in the wear\% is observed for $0.1 \% \mathrm{Cu}$ coated $\mathrm{SiC}$ submicron sized-reinforcement. All nanoreinforced steels showed reduction in the friction coefficient compared to the monolithic steel. The results obtained in this work agree with some previous findings [21-22], suggesting incompatibility between mechanical properties, and wear resistance at high stress wear conditions for nanoreinforced steel. Similar results were observed by the authors on hypoeutectic AISi alloys, but not for hypereutectic AISi alloys [29].

The wear results suggest a stronger effect of the matrix on wear conditions. It is known that the wear resistances of materials are enhanced as their hardness increases (for the same microstructure). It has been shown [28] that the creation of heterogeneous nanostructures is particularly beneficial and has therefore served as an overarching mechanism in promoting strength-ductility synergy. Most of the previous literature and technology innovations were based on enhancing wear resistance via increasing hardness, for the same alloy composition. The concepts of improving wear resistance by modifying microstructures were limited by conventional theories were hardness was usually enhanced by introducing carbides and hard precipitates to the matrix. Enhancing wear resistance by modifying the microstructure (for the same alloy), with little change in hardness will become achievable via nano technology. As the microstructures change to be more resistant to plastic deformation, or as the hardness of the steel increases, less friction coefficients will be induced and the material will become more resistant to wear.

In this work, the steel dispersed with $\mathrm{Cu}$ coated SiC submicronsized particles have shown enhanced friction coefficients compared to both the monolithic and SiC dispersed steels. The discrepancy between wear resistances for different loading conditions is also shown (Figs. 2 and 4). The results suggest that enhancement in wear resistance may be observed at higher loading conditions and longer exposure times. However, these findings only present an early stage of work and still further analysis is needed to identify wear surfaces and boundary morphology between the particles and the matrix. 


\section{CONCLUSIONS}

This work has shown that adding submicron sized-sized $\mathrm{SiC}$ to low $\mathrm{C}$ steel with and without $\mathrm{Cu}$-coating for enhancing wettability resulted a refining effect on the ferrite grain size, but did not result enhancement in the wear resistance of the steel. However, adding the submicron sized particles reduced the friction coefficient of the steel during wear conditions.

\section{REFERENCES}

[1] Hurricks , P, L , : "Some Metallurgical Factors Controlling The Adhesive And Wear Resistance of Steels " , Wear , 26 , 1973 , 285 -304.

[2] Blau , P. J .; "Mechanisms For Transitional Friction And Wear Behavior Of Sliding Metal ", Wear, 72, 1981, 55-66.

[3] Moore, M ,A, :" A review of two body abrasive wear ", Wear, Vol. 27, 1974, 117.

[4] Maroz , P, J. and Lorenzetti , J. J ; "the effects of matrix hardness and microstructure on the wear of steel grinding balls during wet copper ore grinding". Ibid , $280-289$.

[5] khrushov , C, K, and Thomas , G .; " microstructure influence on abrasive wear resistance of high strength, high toughness medium carbon steels ". Conf . Proc. on wear of materials, the American society of mechanical engineers , April 11-14, 1983 , Reston, Virginia , $140-147$

[6] Kar, N. J.; "Investigation Of The Role Of Microstructure On The Two-Body Abrasive Wear Resistance Of Steels." Conf. proc. on wear of materials, the American society of mechanical Engineers, march 30 - April 1, san Francisco, California , $415-425$

[7] Fulcher , J. K ., kosel , T, H, . and fiore , N, F, ; "The Effect Of Carbide Volume Fraction On The Low - Stress Abrasion Resistance Of High Cr-Mo White Cast Irons" Ibid 432 .-438

[8] Zum Gahr , k, H . ; "How Microstructure Affects Abrasive Wear Resistance", Metal Progress", Sep , 1979 , 46 -52

[9] Schey, J, A .; "Metal Deformation Processes, Marcel Dekker, Inc . New York, 1970.

[10] Hurricks , P,L .; " Some Aspects Of The Metallurgy And Wear Resistance Of Surface Coasting" , Wear , 22 , 1972 , PP 291

[11] Wayne. S. F . and Rice, S. L .; "The Role Of Microstructure In The Wear Of Selected Steels", Wear , $85,1983,93-106$

[12] Rice . S. L, ;" Variations In Wear Resistance Due To Microstructural Conditions In High Strength Steel Under Repetitive Impact" , Tribology International, 12 (1), 1979, PP 25.

[13] Nikhilesh Chawla Krishan K. Chawla Metal Matrix Composites Springer 2006 Springer Science+Business Media, Inc. Ch 10: 338-339.

[14] Zuriñe Idoyaga , Lorena M. Callejo and Jacinto Albarrán; Development of new steel grades with improved mechanical properties by the addition of nanoparticles, Workshop on Advanced Steels 2014, Challenges In Steel Science \& Technology, Madrid, 18-19th SEPTEMBER 2014.

[15] El Mahallawi, I.; Shash, Y.; Rashad, R.M.; Abdelaziz, M.H.; Mayer, J.; Schwedt, A. "Hardness and Wear Behavior of Semi-Solid Cast A390 Alloy Reinforced with Al2O3 and TiO2 Nanoparticles", Arab. J. Sci. Eng. 2014, 39, 5171-5184, 
[16] Iman S. El-Mahallawi 1, *, Ahmed Yehia Shash 2, ${ }^{*}$ and Amer Eid Amer; Nanoreinforced Cast Al-Si Alloys with Al2O3, TiO2 and ZrO2Nanoparticles, Metals 2015, 5(2), 802-821; doi:10.3390/met5020802

[17] M.A. Suarez*, M.A. Alvarez-Pérez, O. Alvarez-Fregoso, J.A. Juarez-Islas; Effect of nanoprecipitates and grain size on the mechanical properties of advanced structural steels, Materials Science and Engineering: A Volume 528, Issue 15, 15 June 2011, Pages 4924-4926.

[18] Mohamed Kamal El - Fawkhry, Ahmed Shash, Sherif Ali, Hassan Bahaa and Taha Mattar. Enhancement of pearlitic structure through inoculation with nanosize silicon carbide. Int. J. Nanoparticles, Vol. 7, Nos. 3/4, 2014.

[19] V. I. Kyryliv; Improvement of the wear resistance of medium-carbon steel by nanodispersion of surface layers, Materials Science July 2012, Volume 48, Issue 1, pp 119-123 Date: 22 Aug 2012.

[20] Nanostructured Materials, Processing, Properties, and Applications, edited by Carl C. Koch, Chapter 12, Mechanical Behavior of Nanocrystalline Metals William Andrew Publishing USA, NY USA 2007.

[21] Nanostructured Materials, Processing, Properties and Potential Applications, Edited by Carl C. Koch, NOYES PUBLICATIONS, WILLIAM ANDREW PUBLISHING Norwich, New York, U.S.A. 2002, Chapter 2 Thermal Spray Processing of Nanocrystalline Materials, by Maggy L. Lau and Enrique J. Lavernia

[22] Stewart, D. A., Shipway, P. H., and McCartney, D. G., Abrasive Wear Behaviour of Conventional and Nanocomposite HVOF-sprayed WC-Co coatings, Wear, 225-229:789-798 (1999).

[23] He, J., Ice, M., and Lavernia, E. J., Synthesis of Nanostructured WC-12\%Co Coating Using Mechanical Milling and HVOF Thermal Spraying, Metall. Mater. Trans., 31A:541-553 (2000)

[24] Nanostructured Materials, Processing, Properties, and Applications, edited by Carl C. Koch, Chapter 3, Thermal Sprayed Nanostructured Coatings: Applications and Developments George E. Kim Publishing USA, NY USA 2007.

[25] Riccardo Casati and Maurizio Vedani. Metal Matrix Composites Reinforced by Nano-Particles-A Review. Metals 2014, 4, 65-83.

[26] I. Konyashin, f, B. Ries, D. Hlawatschek, Y. Zhuk, A. Mazilkin, B. Straumal, F. Dorn, D. Park, Wear-resistance and hardness: Are they directly related for nanostructured hard materials?, International Journal of Refractory Metals and Hard Materials, Volume 49, March 2015, Special Issue: International Conference on the Science of Hard Materials - 10, 203-211.

[27] M.A. Suarez*, M.A. Alvarez-Pérez, O. Alvarez-Fregoso, J.A. Juarez-Islas, Effect of nanoprecipitates and grain size on the mechanical properties of advanced structural steels, Materials Science and Engineering A 528 (2011) 4924-4926.

[28] Evan Ma and Ting Zhu, Towards strength-ductility synergy through the design of heterogeneous nanostructures in metals, Materials Today, Volume 20, Number 6 July/August 2017, 323-331.

[29] I.S. El-Mahallawi and A.Y. Shash, Mechanical Properties and Wear Resistance of Semisolid Cast Al2O3 Nano Reinforced Hypo and Hyper-eutectic Al-Si Composites, Properties and Characterization of Modern Materials, Advanced Structured Materials 33, Springer Science+Business Media Singapore 2017, 13-30. 
Figures and Tables

Table 1. Chemical analysis of investigated steel.

\begin{tabular}{|c|c|c|c|c|c|c|}
\hline $\begin{array}{c}\text { Alloy } \\
\text { code }\end{array}$ & C & Mn & Si & Cr & S & P \\
\hline $\mathrm{T}$ & 0.2 & 0.5 & 0.2 & 0.3 & 0.03 & 0.03 \\
\hline
\end{tabular}

Table 2. Content of submicron sized particles.

\begin{tabular}{|c|c|c|c|c|c|c|c|}
\hline & T11 & T12 & T13 & T14 & T21 & T22 & T23 \\
\hline $\begin{array}{c}\text { \% submicron } \\
\text { sized SiC }\end{array}$ & nil & $\begin{array}{c}0.025 \\
\text { SiC }\end{array}$ & $\begin{array}{c}0.05 \\
\text { SiC }\end{array}$ & $\begin{array}{c}0.1 \\
\text { SiC }\end{array}$ & $\begin{array}{c}0.025^{*} \mathrm{Cu}- \\
\text { coated SiC }\end{array}$ & $\begin{array}{c}0.05^{*} \mathrm{Cu}- \\
\text { coated } \\
\text { SiC }\end{array}$ & $\begin{array}{c}0.1^{*} \mathrm{Cu}- \\
\text { coated } \\
\text { SiC }\end{array}$ \\
\hline
\end{tabular}
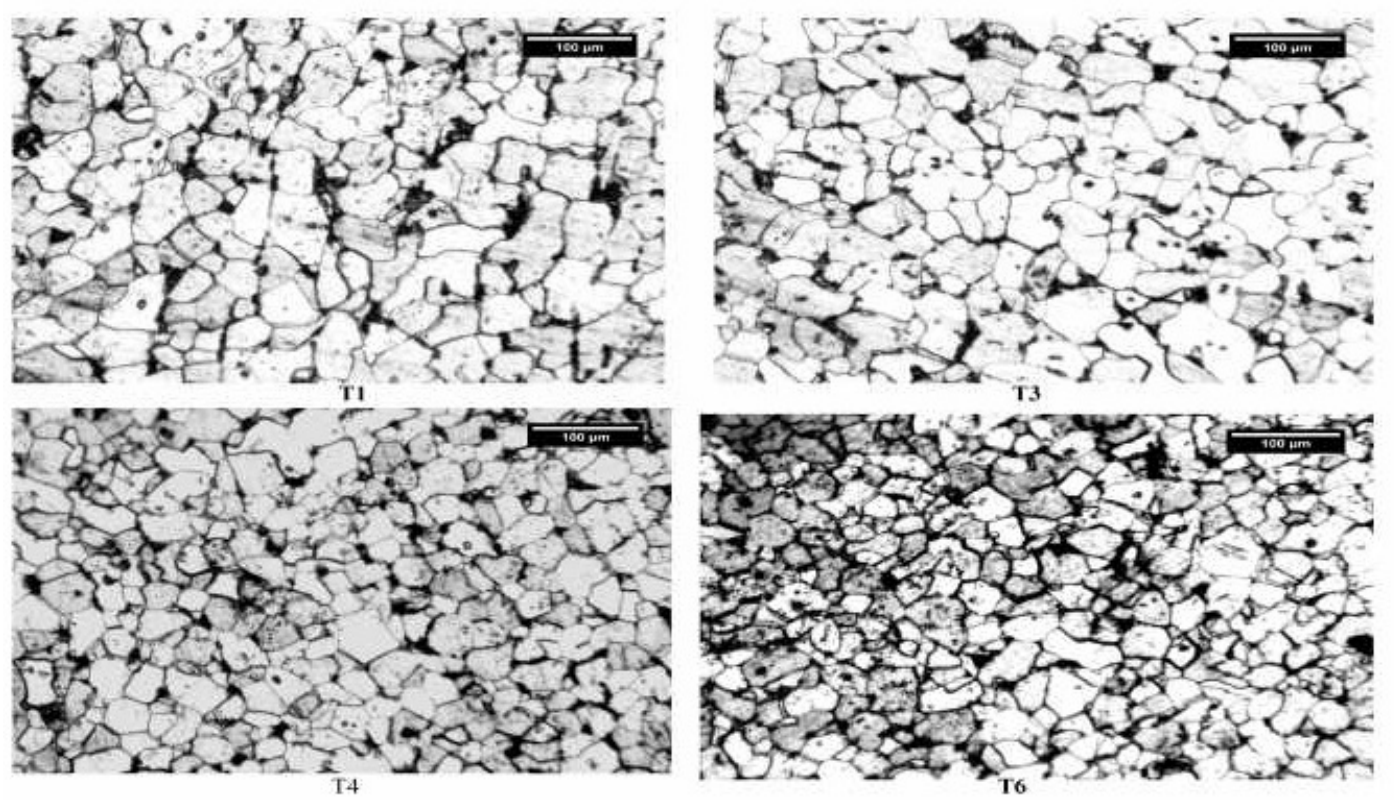

Fig.1 Microstructure of the reference steel $0.2 \% \mathrm{C} \quad 0.5 \% \mathrm{Mn} \quad 0.2 \% \mathrm{Si} 0.3 \% \mathrm{Cr} \quad 0.03 \% \mathrm{~S}$ $0.03 \% \mathrm{P}$ (a) and steel inoculated with $\mathrm{SiC}: 0.05,0.1$ and $0.25 \%$ inoculants(b, c and d); respectively. [18] 


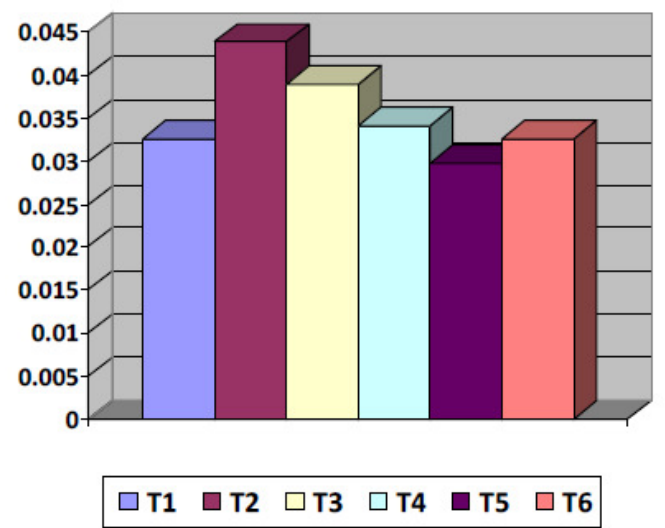

(a)

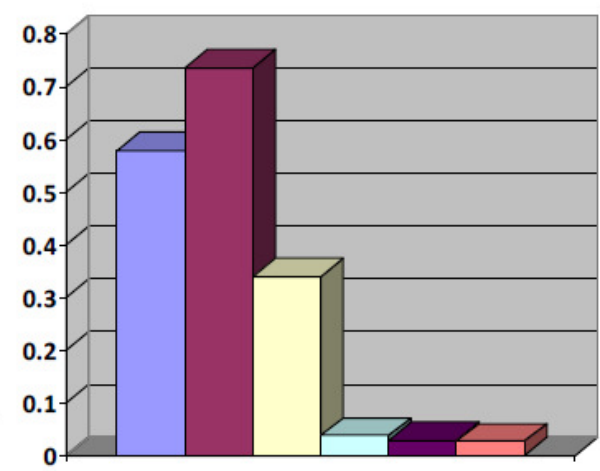

$\square$ T1 $\square$ T2 $\square$ T3 $\square$ T4 $\square$ T5 $\square$ T6

(b)

\begin{tabular}{|c|c|c|c|c|c|c|}
\hline & T1 & T2 & T3 & T4 & T5 & T6 \\
\hline \% submicron sized SiC & 0 & 0.025 & 0.05 & 0.1 & 0.15 & 0.25 \\
\hline
\end{tabular}

Fig. 2 Wear resistance in $\mathrm{g} / \mathrm{min}$ of the investigated steel $0.2 \% \mathrm{C} 0.5 \% \mathrm{Mn} 0.2 \% \mathrm{Si}$ $0.3 \% \mathrm{Cr} 0.03 \% \mathrm{~S} 0.03 \% \mathrm{P}$ at load $0.7 \mathrm{~N}(\mathrm{a}), 0.9 \mathrm{~N}$ (b) [18].

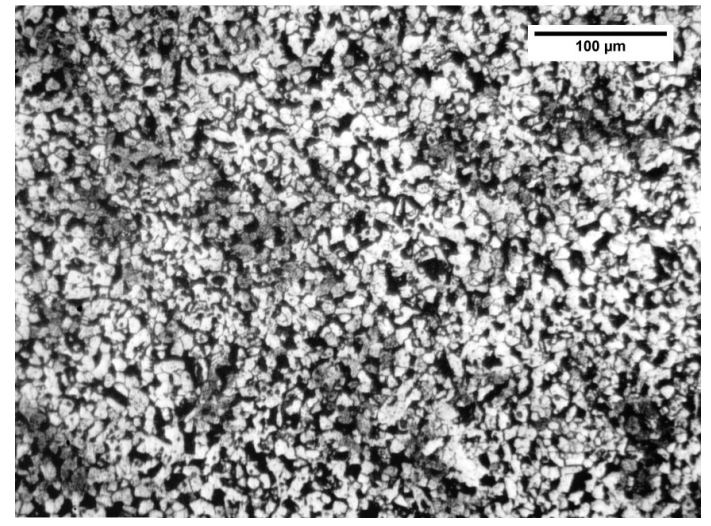

a) T11

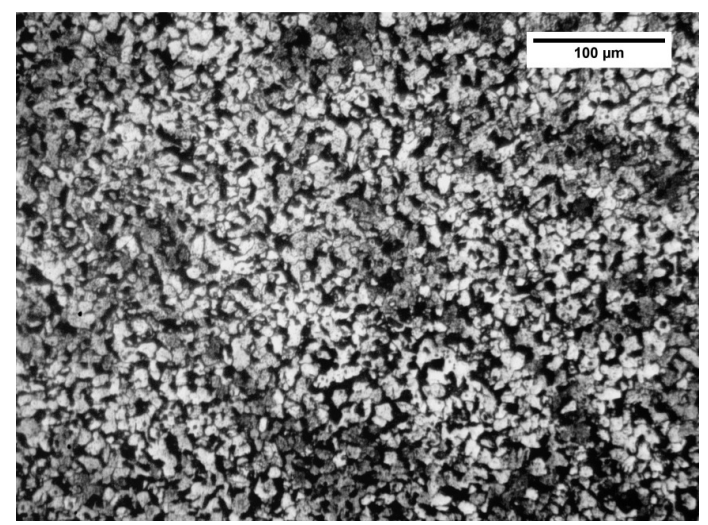

c) T21

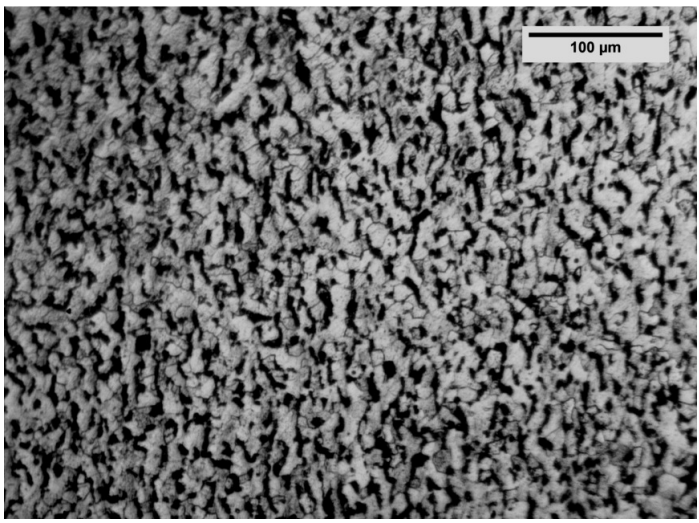

b) T13

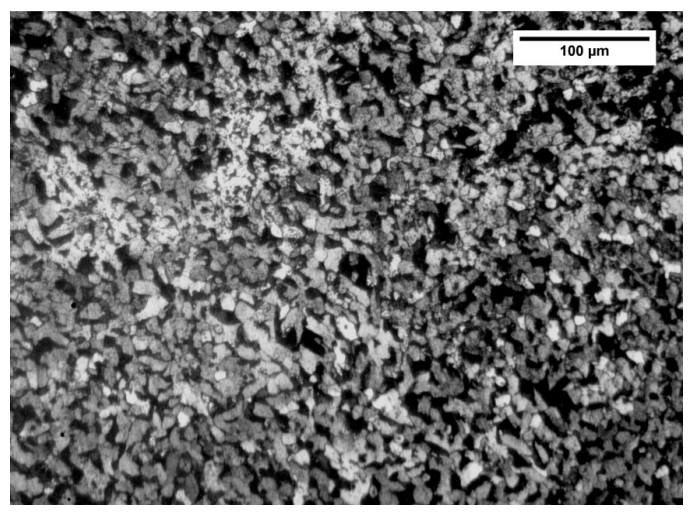

d) T23

Fig. 3 Microstructure of the $\mathrm{SiC}$ reinforced steel (a and b) and the Cu-coated $\mathrm{SiC}$ reinforced steel (c and d). 

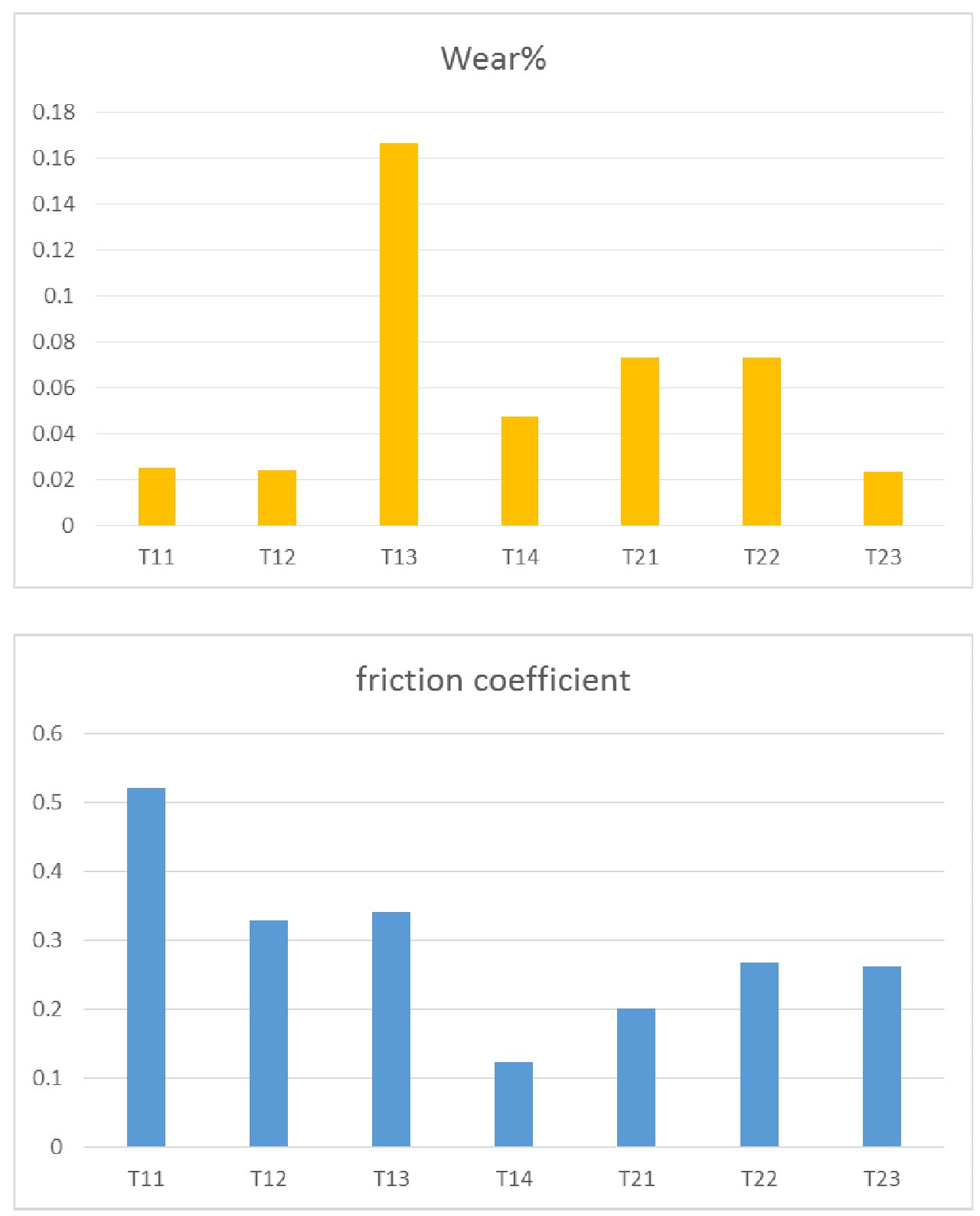

\begin{tabular}{|c|c|c|c|c|c|c|c|}
\hline & T11 & T12 & T13 & T14 & T21 & T22 & T23 \\
\hline $\begin{array}{c}\text { \% submicron } \\
\text { sized SiC }\end{array}$ & 0 & 0.025 & 0.05 & 0.1 & $0.025^{\star}$ & $0.05^{*}$ & $0.1^{*}$ \\
\hline
\end{tabular}

* SiC submicron sized particles were mechanically coated with copper

Fig. 4. Wear\% results using Pin-on-disc tester, 5N. 